\title{
Epistemic beliefs as a determinant in evidence-based practice in physiotherapy - a multi-country (Europe) cross-sectional online survey study
}

\author{
Paul Christiaan Beenen ${ }^{\mathrm{a}, \mathrm{b}}$, Dario Filiputti ${ }^{\mathrm{c}}$, Erna Rosenlund Meyer ${ }^{\mathrm{d}}$, Lidia \\ Carballo-Costa ${ }^{\mathrm{e}}$, Patricia Maria Duarte de Almeida ${ }^{\mathrm{a}, \mathrm{f}}$, Antonio Alves Lopes ${ }^{\mathrm{f}}$, Joost \\ Egbertus Jacobus Lidwina van Wijchen ${ }^{\mathrm{g}}$ and Alexandre Castro Caldas ${ }^{\mathrm{b}}$
}

\footnotetext{
${ }^{a}$ Hanze University of Applied Sciences, Groningen, The Netherlands;

${ }^{b}$ Institute of Health Sciences, Catholic University of Portugal, Lisbon, Portugal;

${ }^{c}$ Department of Medical, Experimental and Clinical Sciences, University of Udine, Udine, Italy;

${ }^{d}$ Department of Physiotherapy, University College of Northern Denmark, Aalborg, Denmark;

${ }^{e}$ Department of Biomedical Sciences, Medicine and Physiotherapy, Grupo de investigación de Intervención Psicosocial y Rehabilitación funcional, Universidade da Coruña (University of A Coruña), A Coruña, Spain; ${ }^{f}$ Department of Physiotherapy, Alcoitao School of Health Sciences, Alcoitao, Portugal;

${ }^{g}$ Department of Physiotherapy, HAN University of Applied Sciences, Nijmegen, The Netherlands
}

\begin{abstract}
Purpose: This article assumes that, in order to improve evidence-based practice in physiotherapy, practitioners need sophisticated epistemic beliefs. Epistemic beliefs, or how physiotherapists view knowledge and how they come to this knowledge, are an important factor. A high sophistication of epistemic beliefs is linked to better handling of the complexity and uncertainty of daily practice and the variety of evidence resources associated with this practice.

Materials and methods: This study explored the epistemic beliefs of physiotherapists in 10 different countries in Europe using an online survey: the Connotative Aspects of Epistemic Beliefs (CAEB).

Results and conclusions: The study resulted in 1419 surveys. The epistemic beliefs proved to be of little difference between countries, showing a low to moderate sophistication in epistemic beliefs. Given the similar results between countries, this study also suggested the possibility of collaborating internationally in developing an epistemology in physiotherapy that is more suited to the complexity of current demands on health systems. The development of sophistication in epistemic beliefs should be firmly on the agenda for the education of physiotherapists.
\end{abstract}

Keywords: Epistemic beliefs, epistemological beliefs, physiotherapy, evidence-based practice 


\section{Introduction}

This article reports on a research of the epistemic beliefs of physiotherapists throughout the community of physiotherapy in Europe. Epistemic beliefs are about focussing on what individuals believe concerning what counts as knowledge and where it resides, how individuals come to know, and how knowledge is constructed and evaluated [1,2]. These beliefs influence the way an individual evaluates new information. How an individual resolves conflicting knowledge claims, makes decisions, and thus is able to work in an evidence-based manner [3,4]. Epistemic beliefs determine how (new) knowledge is perceived and processed [5]. Though this is a major issue in evidence-based practice in health care, there has been little research on epistemic beliefs in physiotherapy. Therefore, the article begins with a theoretical background to explain the concept of epistemic beliefs in relation to physiotherapy practice.

\section{Epistemic beliefs in decision making}

Within the model of evidence-based practice, the beliefs of what physiotherapists consider adequate knowledge and how they acquire this knowledge are important factors. Epistemic beliefs can be considered as a focal point for how physiotherapists create meaning in their daily practice, what their strategies are for selecting knowledge, what is relevant for decision-making, how this affects the ongoing learning process of accumulating experiences among individual physiotherapists, and how this has its place within the professional community. Epistemic beliefs can be placed within a spectrum. At one end, we have a naïve view that knowledge comes from an authority or scientific source and is objective and static by nature. At the other end, we have the 'sophisticated' view that knowledge is only valid within the specific professional situation. Knowledge in this sense is inherently dynamic and mutable and needs constant scrutiny. In the naïve view, the physiotherapist is likely to take a position as the authoritative expert-professional using 'objective' acquired knowledge that is deemed to be true at all times. In the sophisticated view, the relation with the client and other resources will be much more important to come to a collective diagnosis.

Epistemic beliefs directly influence decision-making. The naïve understanding of knowledge assumes transmission from an authority to the learner (from teacher to physiotherapist or from physiotherapist to client). Individuals justify what they know and how they evaluate their knowledge based on factual generalisations. This originates from the 'naïve' belief that (scientific) authority, or 'what feels right' is also valid. The 'sophisticated' beliefs, on the other hand, maintain that knowledge can be justified by evaluating a diversity of sources of evidence, including patient values, expertise, scientific evidence and context-based factors within each and every specific situation. Such beliefs lead, in practice, to the use of more constructivist and more self-regulated learning [6].

\section{Research epistemic beliefs}

Little specific research has been done in epistemic beliefs in physiotherapy education or practice [7]. Most research in epistemic beliefs shares the view that epistemological knowledge consists of declarative beliefs that can be articulated by the individual [1]. This view is challenged by another viewpoint, which argues that many beliefs are implicit and thus less articulated [8].

Explicit knowledge refers, in this perspective, to the concept of knowledge for those practices that are relatively prescriptive, such as the framework of evidence-based practice, guidelines in physiotherapy or the role of science. A physiotherapist would reflect explicitly on what value he gives to the role of, for example, a guideline.

The other perspective is a more implicit concept of knowledge of the individual professional arising directly from the complexity of daily practice, which is much more personal, emotional and context-dependent [9]. This way of determining beliefs includes not only the explicit rationalities, 
often conditioned by education, but also the more implicit and personal preference for knowledge in the complexity of real practice $[10,11]$.

Stahl and Bromme developed a new instrument for measuring Connotative Aspects of Epistemic Beliefs: the CAEB [8]. Connotative meanings refer to associative and evaluative judgments. The term comes from linguistics, where it refers to additional and individual meanings that a person associates with a concept or word. The CAEB uses a semantic-differential scale with opposite adjectives with a seven-point Likert scale. These adjectives, for the purpose of this study, are meant to analyse how physiotherapists perceive physiotherapy-related knowledge. The questionnaire shows two dimensions: texture and variability. The dimension of texture is defined as beliefs about the structure and accuracy of knowledge. This dimension ranges from the belief that knowledge is exact and structured to the belief that it is unstructured and vague. The dimension of variability is defined as beliefs about the stability and dynamics of knowledge. This dimension ranges from the belief that knowledge is stable and inflexible, to the belief that it is dynamic and flexible.

The above research suggests that a physiotherapist benefits from sophisticated epistemic beliefs in order to act in accordance with the challenges of the evidence-based movement [12]. The complex nature of physiotherapy demands a view of scientific knowledge as a coherent, hierarchical system of ideas, rather than as a simple collection of facts. The complexity and uncertainty that physiotherapists are facing in their daily practice demands the need to have the skills to approach these ill-structured problems in a more active and critical manner. This is associated with progression, or sophistication, in epistemic thinking towards a higher level $[13,14]$.

Physiotherapy represents a strong international community and actively crosses borders to related scientific communities. In this 'knowledge society', one of the goals is to understand and jointly develop the profession and relevant models of practice, such as evidence-based practice [15].

This study explores the level of sophistication of epistemic beliefs of the community of physiotherapists within Europe, answering the following research questions: how sophisticated are the epistemic beliefs of physiotherapists; do epistemic beliefs differ among physiotherapists with regard to their level of education, years of experience, gender and country?

\section{Methods}

An online survey study was implemented in 10 countries using the Connotative Aspects of Epistemological Beliefs (CAEB) [8]. This questionnaire was specifically adapted for the countries and their languages in this study, showing satisfactory validity in the context of physiotherapy [16]. The CAEB uses a semantic-differential scale with opposite adjectives with a seven-point Likert scale (see Table 1 for the adjectives and supplementary material 1 for the English version of the CAEB. The CAEB questionnaire was used with permission, see supplementary material 2). 
Table 1. CAEB - Connotative Aspects of Epistemological Beliefs.

\begin{tabular}{ll}
\hline Items & $\begin{array}{l}\text { Factors Stahl } \\
\mathrm{R}=\text { reversed }\end{array}$ \\
\hline & \\
1. Stable-Instable & Variability \\
2. Objective-Subjective & Texture \\
3. Confirmable-Unconfirmable & Texture \\
4. Dynamic-static & Texture (R) \\
5. Superficial-profound & Texture \\
6. Temporary-everlasting & Variability (R) \\
7. Exact-vague & Texture \\
8. Absolute-Relative & Texture \\
9. Sorted-Unsorted & Texture \\
10. Precise-Imprecise & Texture \\
11. Flexible-Inflexible & Variability (R) \\
12. Definite-Ambiguous & Texture \\
13. Negotiated-Discovered & Texture (R) \\
14. Structured-Unstructured & Texture \\
15. Completed-Uncompleted & Variability \\
16. Refutable-Irrefutable & Variability (R) \\
17. Open-Closed & Variability (R) \\
\hline
\end{tabular}

The CAEB questionnaire is used with the permission of the authors and was first published in [8]. See for the questionnaire also the supplementary material 1 .

\section{Subjects and data acquisition}

Aiming to represent the European community of physiotherapy, we managed to obtain the collaboration of 10 countries (out of 36). Within these countries we collected data from physiotherapy practitioners, teachers and students. This was done using the 'European Network of Physiotherapy in Higher Education', based on the interest of the members and the possibility of investing time (ENPHE). The following countries were included; Finland, Sweden, Denmark, the Netherlands, Italy, Spain, Portugal, Germany, Austria and Switzerland. The last three countries were treated as one region (German Speaking Countries: GSC) as they showed similar results in the adaptation process of the questionnaire [16]. More detailed characteristics for the total sample and per country are presented in Table 2 . 
Table 2. Sample characteristics

\begin{tabular}{|c|c|c|c|c|c|c|c|c|c|c|}
\hline & & Netherlands & Portugal & Denmark & Italy & Spain & Finland & $\mathrm{GSC}^{\mathrm{a}}$ & Sweden & Total \\
\hline$N$ & & 283 & 277 & 151 & 218 & 229 & 105 & 123 & 33 & 1419 \\
\hline \multirow[t]{2}{*}{ Percentage of male and female } & Female & 51.2 & 76.5 & 69.5 & 58.3 & 68.1 & 81.0 & 77.2 & 86.7 & 67.2 \\
\hline & Male & 48.8 & 23.5 & 30.5 & 41.7 & 31.9 & 19.0 & 22.8 & 13.3 & 32.8 \\
\hline \multirow[t]{4}{*}{$\begin{array}{l}\text { Percentage of the age groups } \\
\text { (given in years) }\end{array}$} & $20-29$ & 32.9 & 41.9 & 37.7 & 33.0 & 54.6 & 41.9 & 56.9 & 30.0 & 41.5 \\
\hline & $30-39$ & 24.7 & 31.4 & 21.2 & 20.2 & 31.9 & 21.9 & 18.7 & 23.3 & 25.3 \\
\hline & $40-49$ & 14.5 & 13.0 & 16.6 & 16.1 & 9.6 & 11.4 & 11.4 & 23.3 & 13.5 \\
\hline & $>50$ & 27.9 & 13.7 & 24.5 & 30.7 & 3.9 & 24.8 & 13 & 23.3 & 19.7 \\
\hline \multirow[t]{4}{*}{$\begin{array}{l}\text { Percentage of groups } \\
\text { represented in years of licence }\end{array}$} & $<5$ & 27.6 & 30.7 & 39.1 & 36.2 & 50.2 & 53.3 & 52 & 36.7 & 38.6 \\
\hline & $5-10$ & 20.8 & 35.7 & 12.6 & 8.7 & 14.8 & 10.5 & 17.9 & 16.7 & 18.9 \\
\hline & $11-15$ & 8.5 & 11.9 & 12.6 & 10.6 & 17.9 & 5.7 & 6.5 & 10.0 & 11.1 \\
\hline & $>15$ & 43.1 & 21.7 & 35.8 & 44.5 & 17.0 & 30.5 & 23.6 & 36.7 & 31.4 \\
\hline \multirow[t]{7}{*}{$\begin{array}{l}\text { Percentage of groups } \\
\text { represented by educational } \\
\text { level }\end{array}$} & $\begin{array}{l}\text { Prof. } \\
\text { diploma }\end{array}$ & - & - & - & - & - & - & 20.3 & 6.7 & 1.9 \\
\hline & $\begin{array}{l}\text { Bachelor } \\
\text { student }\end{array}$ & 13.4 & 11.2 & 29.9 & 50.5 & 34.1 & 39.0 & 41.5 & 10.0 & 22.6 \\
\hline & $\begin{array}{l}\text { Bachelor } \\
\text { degree }\end{array}$ & 50.4 & 62.1 & 59.6 & 22.5 & 35.4 & 33.3 & 18.7 & 40.0 & 47.4 \\
\hline & $\begin{array}{l}\text { Master } \\
\text { student }\end{array}$ & 8.8 & 5.8 & 05.3 & 1.8 & 5.2 & 3.8 & 5.7 & 6.7 & 5.5 \\
\hline & $\begin{array}{l}\text { Master } \\
\text { degree }\end{array}$ & 20.1 & 15.5 & 12.6 & 24.8 & 13.5 & 15.2 & 10.6 & 10.0 & 16.6 \\
\hline & PhD student & 3.5 & 2.9 & 0.7 & 0 & 6.6 & 4.8 & 3.3 & 10.0 & 3.2 \\
\hline & PhD degree & 3.5 & 2.5 & 0 & 0.5 & 5.2 & 3.8 & 0 & 16.7 & 2.7 \\
\hline
\end{tabular}

${ }^{a}$ GSC: German Speaking Countries.

The questionnaire per country was constructed on Google Drive forms with the automatic creation of an Excel database for data recording. The sample acquisition varied among countries through the use of mailing lists from educational institutes and professional associations.

\section{Data analysis}

The data recorded on the excel databases (per country) were exported to a single database created on the SPSS ${ }^{\circledR}$ version 22 (SPSS Inc., Chicago, IL) for the statistical analysis.

\section{Validity of the CAEB}

Analysis of the internal consistency to assess reliability and a factor analysis were performed, with the two factors described in the original studies [8], to confirm the construct validity. Based on the rule that the initial eigenvalues should be $>1$, a minimum of the proposed factors was recognised in all countries, allowing for a factor analysis. Solutions were confirmed by successively omitting items with no substantial factor loadings (<.32) [17]. Items were also omitted with high loadings ( $>.40)$ on more than one factor [11]. In order to compare and correlate the scores, an inversion of the Likert scale was performed with 'recode into same variables' within SPSS-22 to align the scores towards the same direction on the spectrum of naive to sophisticated epistemic beliefs. The following items were inverted: 4, 6, 11, 13, 16 and 17 (see Table 1). 
To detect the sophistication of epistemic beliefs in the sample, a descriptive analysis was performed. This was done by means of a mean, standard deviation and the minimum and maximum per admitted factor calculated for all countries and for the general characteristics.

Analysis of variance

For the comparison between dependent variables (scores of the CAEB) and independent variables (level of education, years of experience, gender and country), a one-way MANOVA was performed, considering $p$ values $<.05$ for statistical significance.

\section{Results}

\section{Validity of the $C A E B$}

The normality of the sample was confirmed with the Shapiro-Wilk test. The Measure of Sample Adequacy (MSA) that reports the appropriateness of data for a factor analysis was confirmed. According to [18], the MSA is satisfactory with values >.80 (18). The MSA values showed acceptable values $(.890-p \leq .000)$.

The factor analysis was showing acceptable Cronbach's values, for both the total questionnaire and for the factors (Tables 3 and 4). The results offer sufficient validity to allow for interpretation of the results from the sample. 
Table 3. Results factor analysis CAEB.

\begin{tabular}{ll}
\hline & \multicolumn{2}{c}{ Factors } \\
\cline { 2 - 3 } Item & Texture $\quad$ Variability
\end{tabular}

\begin{tabular}{|c|c|c|}
\hline 1 & .571 & .104 \\
\hline 2 & .608 & -.163 \\
\hline 3 & .543 & -.325 \\
\hline 4 & -.229 & .722 \\
\hline $5^{*}$ & .052 & -.121 \\
\hline 6 & .064 & .579 \\
\hline 7 & .711 & -.204 \\
\hline 8 & .648 & .227 \\
\hline 9 & .718 & .152 \\
\hline 10 & .761 & -.192 \\
\hline 11 & .276 & .716 \\
\hline 12 & .715 & -.023 \\
\hline $13^{*}$ & -.292 & .110 \\
\hline 14 & .664 & -.272 \\
\hline 15 & .687 & .117 \\
\hline 16 & -.079 & .680 \\
\hline 17 & -.310 & .712 \\
\hline Cronbach & .862 & .762 \\
\hline
\end{tabular}

Cronbach of the validated questionnaire: .840

Cronbach with omitted items: .853

Total variance explained: $44,1 \%$

Numbers with an * were omitted due to low loading $<.320$
Table 4. Organisation of the CAEB items per factors/dimensions.

\begin{tabular}{ll}
\hline Item & Description \\
\hline & \\
\multicolumn{2}{l}{ Factor/dimension - Texture } \\
1 & Stable-instable \\
2 & Objective-Subjective \\
3 & Confirmable-Unconfirmable \\
7 & Exact-vague \\
8 & Absolute-Relative \\
9 & Sorted-Unsorted \\
10 & Precise-Imprecise \\
12 & Definite-Ambiguous \\
14 & Structured-Unstructured \\
15 & Completed-Uncompleted \\
Factor/dimension - Variability \\
4 & Dynamic-static \\
6 & Temporary-everlasting \\
11 & Flexible-Inflexible \\
16 & Refutable-Irrefutable \\
17 & Open-Closed \\
\end{tabular}

\section{Sophistication of epistemic beliefs}

The CAEB factor texture has a mean score $=39.17 \pm 9.29$, given the possible range from minimum 10 to maximum 70 . This corresponds to a below medium level of sophisticated beliefs for the total sample. The physiotherapists in the sample perceive knowledge in the field of physiotherapy at just below the middle of the spectrum ranging from naïve belief to sophisticated belief. The scores are presented in Table 5 . 
Table 6. Descriptive statistics CAEB variability

\begin{tabular}{|c|c|c|c|c|c|}
\hline & $N$ & Minimum & Maximum & $\begin{array}{l}\text { Mean } \\
\text { score }\end{array}$ & $\begin{array}{c}\text { Std. } \\
\text { deviation }\end{array}$ \\
\hline Total & 1419 & 5.00 & 35.00 & 15.23 & 5.36 \\
\hline \multicolumn{6}{|l|}{ Per nationality } \\
\hline The Netherlands & 283 & 5.00 & 34.00 & 14.93 & 4.89 \\
\hline Portugal & 277 & 5.00 & 33.00 & 16.35 & 6.16 \\
\hline Denmark & 151 & 7.00 & 35.00 & 16.53 & 5.28 \\
\hline Italy & 218 & 5.00 & 35.00 & 14.65 & 5.32 \\
\hline Spain & 229 & 5.00 & 35.00 & 13.13 & 5.11 \\
\hline Finland & 105 & 8.00 & 28.00 & 16.16 & 3.95 \\
\hline GSC & 123 & 5.00 & 32.00 & 15.95 & 4.95 \\
\hline Sweden & 33 & 8.00 & 26.00 & 15.27 & 4.80 \\
\hline \multicolumn{6}{|l|}{ Per gender } \\
\hline Female & 954 & 5.00 & 35.00 & 15.34 & 5.45 \\
\hline Male & 456 & 5.00 & 34.00 & 15.01 & 5.17 \\
\hline \multicolumn{6}{|l|}{ Per age range } \\
\hline $20-29$ & 589 & 5.00 & 32.00 & 14.40 & 4.94 \\
\hline $30-39$ & 359 & 5.00 & 35.00 & 15.54 & 5.63 \\
\hline $40-49$ & 192 & 5.00 & 35.00 & 15.67 & 5.46 \\
\hline$>50$ & 279 & 5.00 & 35.00 & 16.29 & 5.54 \\
\hline \multicolumn{6}{|l|}{ Per level of education } \\
\hline Professional diploma & 27 & 5.00 & 29.00 & 15.74 & 4.85 \\
\hline Bachelor student & 321 & 5.00 & 32.00 & 14.72 & 4.78 \\
\hline Bachelor & 672 & 5.00 & 35.00 & 15.35 & 5.50 \\
\hline Master student & 78 & 5.00 & 32.00 & 15.87 & 5.81 \\
\hline Master & 236 & 5.00 & 33.00 & 15.37 & 5.42 \\
\hline $\mathrm{PhD}$ student & 46 & 5.00 & 34.00 & 16.11 & 6.75 \\
\hline $\mathrm{PhD}$ & 39 & 7.00 & 23.00 & 13.92 & 4.40 \\
\hline \multicolumn{6}{|l|}{ Licensed years } \\
\hline$<5$ & 255 & 5.00 & 35.00 & 15.41 & 5.78 \\
\hline $5-10$ & 185 & 5.00 & 35.00 & 14.98 & 5.33 \\
\hline $11-15$ & 91 & 6.00 & 34.00 & 14.61 & 4.73 \\
\hline$>15$ & 273 & 5.00 & 33.00 & 15.38 & 5.04 \\
\hline
\end{tabular}

The CAEB factor variability has a mean score of $15.23 \pm 5.36$, given the possible range of minimum 5 and maximum 35. This corresponds to a low level of sophisticated beliefs for the total sample. On a spectrum ranging from naïve belief to sophisticated belief, the sample scores quite low. The scores are presented in Table 6. 
Table 6. Descriptive statistics CAEB variability

\begin{tabular}{|c|c|c|c|c|c|}
\hline & $N$ & Minimum & Maximum & $\begin{array}{c}\text { Mean } \\
\text { score }\end{array}$ & $\begin{array}{c}\text { Std } \\
\text {. deviation }\end{array}$ \\
\hline Total & 1419 & 5.00 & 35.00 & 15.23 & 5.36 \\
\hline \multicolumn{6}{|l|}{ Per nationality } \\
\hline The Netherlands & 283 & 5.00 & 34.00 & 14.93 & 4.89 \\
\hline Portugal & 277 & 5.00 & 33.00 & 16.35 & 6.16 \\
\hline Denmark & 151 & 7.00 & 35.00 & 16.53 & 5.28 \\
\hline Italy & 218 & 5.00 & 35.00 & 14.65 & 5.32 \\
\hline Spain & 229 & 5.00 & 35.00 & 13.13 & 5.11 \\
\hline Finland & 105 & 8.00 & 28.00 & 16.16 & 3.95 \\
\hline GSC & 123 & 5.00 & 32.00 & 15.95 & 4.95 \\
\hline Sweden & 33 & 8.00 & 26.00 & 15.27 & 4.80 \\
\hline \multicolumn{6}{|l|}{ Per gender } \\
\hline Female & 954 & 5.00 & 35.00 & 15.34 & 5.45 \\
\hline Male & 456 & 5.00 & 34.00 & 15.01 & 5.17 \\
\hline \multicolumn{6}{|l|}{ Per age range } \\
\hline $20-29$ & 589 & 5.00 & 32.00 & 14.40 & 4.94 \\
\hline $30-39$ & 359 & 5.00 & 35.00 & 15.54 & 5.63 \\
\hline $40-49$ & 192 & 5.00 & 35.00 & 15.67 & 5.46 \\
\hline$>50$ & 279 & 5.00 & 35.00 & 16.29 & 5.54 \\
\hline \multicolumn{6}{|l|}{ Per level of education } \\
\hline Professional diploma & 27 & 5.00 & 29.00 & 15.74 & 4.85 \\
\hline Bachelor student & 321 & 5.00 & 32.00 & 14.72 & 4.78 \\
\hline Bachelor & 672 & 5.00 & 35.00 & 15.35 & 5.50 \\
\hline Master student & 78 & 5.00 & 32.00 & 15.87 & 5.81 \\
\hline Master & 236 & 5.00 & 33.00 & 15.37 & 5.42 \\
\hline PhD student & 46 & 5.00 & 34.00 & 16.11 & 6.75 \\
\hline $\mathrm{PhD}$ & 39 & 7.00 & 23.00 & 13.92 & 4.40 \\
\hline \multicolumn{6}{|l|}{ Licensed years } \\
\hline$<5$ & 255 & 5.00 & 35.00 & 15.41 & 5.78 \\
\hline $5-10$ & 185 & 5.00 & 35.00 & 14.98 & 5.33 \\
\hline $11-15$ & 91 & 6.00 & 34.00 & 14.61 & 4.73 \\
\hline$>15$ & 273 & 5.00 & 33.00 & 15.38 & 5.04 \\
\hline
\end{tabular}

Differences in epistemic beliefs within the sample

In comparison with general characteristics, the following significant differences are found in the sample: 
Nationality. Statistically significant differences were found for several countries. The northern and Central-European countries often, though not consistently, scored significantly higher in sophistication than the southern countries (Table 7).

Table 7. MANOVA test results for nationality

\begin{tabular}{|c|c|c|c|c|c|c|}
\hline & \multicolumn{2}{|c|}{$\mathrm{CAEB}^{\mathrm{a}}$-total } & \multicolumn{2}{|c|}{ CAEB-text } & \multicolumn{2}{|c|}{ CAEB-variability } \\
\hline & $\mathrm{MD}^{\mathrm{b}}$ & $\mathrm{Sig}^{\mathrm{b}}$ & MD & Sig & MD & Sig \\
\hline \multicolumn{7}{|l|}{ Netherlands } \\
\hline Portugal & 2.14 & 0.474 & 3.70 & 0.000 & -1.41 & 0.032 \\
\hline Spain & 5.95 & 0.000 & 3.61 & 0.000 & 1.81 & 0.003 \\
\hline Denmark & -2.01 & 0.759 & -0.41 & 1.000 & -1.60 & 0.052 \\
\hline Italy & 1.81 & 0.751 & 1.08 & 0.894 & 0.29 & 0.999 \\
\hline Finland & -0.17 & 1.000 & 1.20 & 0.945 & -1.23 & 0.448 \\
\hline Sweden & 1.47 & 0.998 & 1.69 & 0.974 & -0.34 & 1.000 \\
\hline $\mathrm{GSC}^{\mathrm{b}}$ & -1.50 & 0.956 & -1.03 & 0.967 & -1.02 & 0.622 \\
\hline \multicolumn{7}{|l|}{ Portugal } \\
\hline Spain & 3.81 & 0.016 & -0.09 & 1.000 & 3.22 & 0.000 \\
\hline Denmark & -4.14 & 0.025 & -4.10 & 0.000 & -0.18 & 1.000 \\
\hline Italy & -0.33 & 1.000 & -2.62 & 0.035 & 1.70 & 0.009 \\
\hline Finland & -2.31 & 0.748 & -2.49 & 0.252 & 0.18 & 1.000 \\
\hline Sweden & -0.66 & 1.000 & -2.00 & 0.935 & 1.07 & 0.954 \\
\hline GSC & -3.63 & 0.133 & -4.73 & 0.000 & 0.40 & 0.997 \\
\hline \multicolumn{7}{|l|}{ Spain } \\
\hline Denmark & -7.96 & 0.000 & -4.01 & 0.001 & -3.40 & 0.000 \\
\hline Italy & -4.14 & 0.012 & -2.52 & 0.070 & -1.52 & 0.046 \\
\hline Finland & -6.12 & 0.001 & -2.40 & 0.335 & -3.04 & 0.000 \\
\hline Sweden & -4.48 & 0.541 & -1.91 & 0.952 & -2.15 & 0.354 \\
\hline GSC & -7.45 & 0.000 & -4.64 & 0.000 & -2.82 & 0.000 \\
\hline \multicolumn{7}{|l|}{ Denmark } \\
\hline Italy & 3.82 & 0.079 & 1.49 & 0.787 & 1.88 & 0.016 \\
\hline Finland & 1.84 & 0.945 & 1.61 & 0.864 & 0.37 & 0.999 \\
\hline Sweden & 3.48 & 0.838 & 2.10 & 0.933 & 1.26 & 0.918 \\
\hline GSC & 0.51 & 1.000 & -0.63 & 0.999 & 0.58 & 0.985 \\
\hline \multicolumn{7}{|l|}{ Italy } \\
\hline Finland & -1.98 & 0.888 & 0.12 & 1.000 & -1.52 & 0.227 \\
\hline Sweden & -0.34 & 1.000 & 0.61 & 1.000 & -0.63 & 0.998 \\
\hline GSC & -3.31 & 0.275 & -2.12 & 0.447 & -1.30 & 0.349 \\
\hline \multicolumn{7}{|l|}{ Finland } \\
\hline Sweden & 1.64 & 0.998 & 0.49 & 1.000 & 0.89 & 0.990 \\
\hline GSC & -1.33 & 0.993 & -2.24 & 0.591 & 0.21 & 1.000 \\
\hline \multicolumn{7}{|l|}{ Sweden } \\
\hline GSC & -2.97 & 0.930 & -2.73 & 0.796 & -0.68 & 0.998 \\
\hline
\end{tabular}

${ }^{a}$ CAEB: Connotative Aspects of Epistemological Beliefs.

${ }^{\mathrm{b}} \mathrm{MD}$ : mean difference; Sig: significance ( $p$ value); GSC: German speaking countries. 
Age. The 20-29 years old age group scored significantly lower in the total score compared to physiotherapists of 50 years and older. The 20-29 years old age group scored significantly lower than all other age groups compared to the factor variability (Table 8).

Table 8. MANOVA test results for age in CAEB

\begin{tabular}{|c|c|c|c|c|c|c|}
\hline & \multicolumn{2}{|c|}{$\mathrm{CAEB}^{\mathrm{a}}$-total } & \multicolumn{2}{|c|}{ CAEB-text } & \multicolumn{2}{|c|}{ CAEB-variability } \\
\hline & $\mathrm{MD}^{\mathrm{b}}$ & $\mathrm{Sig}^{\mathrm{b}}$ & $\mathrm{MD}$ & $\operatorname{sig}$ & $\mathrm{MD}$ & sig \\
\hline \multicolumn{7}{|l|}{$20-29$} \\
\hline $20-39$ & -0.82 & 0.770 & 0.18 & 0.992 & -1.14 & 0.007 \\
\hline $40-49$ & -1.55 & 0.458 & -0.30 & 0.980 & -1.27 & 0.021 \\
\hline$>50$ & -2.39 & 0.049 & -0.64 & 0.780 & -1.90 & 0.000 \\
\hline \multicolumn{7}{|l|}{$20-39$} \\
\hline $40-49$ & -0.73 & 0.918 & -0.48 & 0.938 & 1.14 & 0.007 \\
\hline$>50$ & -1.57 & 0.414 & -0.82 & 0.688 & -0.13 & 0.993 \\
\hline \multicolumn{7}{|l|}{$40-49$} \\
\hline$>50$ & -0.83 & 0.898 & -0.34 & 0.981 & -0.63 & 0.589 \\
\hline
\end{tabular}

${ }^{a}$ CAEB: Connotative Aspects of Epistemological Beliefs.

${ }^{\mathrm{b}} \mathrm{MD}$ : mean difference; Sig: significance ( $p$ value). 
Level of education. Significant differences were found in the CAEB texture, showing master students having a higher score than physiotherapists with a Bachelor diploma (MD $2.3 ; p \leq .04$ ) (Table 9).

Table 9. MANOVA test results for education.

\begin{tabular}{|c|c|c|c|c|c|c|}
\hline & \multicolumn{2}{|c|}{$\mathrm{CAEB}^{\mathrm{a}}$-total } & \multicolumn{2}{|c|}{ CAEB-text } & \multicolumn{2}{|c|}{ CAEB-variability } \\
\hline & $\mathrm{MD}^{\mathrm{b}}$ & $\operatorname{Sig}^{b}$ & $\mathrm{MD}$ & Sig & MD & Sig \\
\hline \multicolumn{7}{|c|}{ Professional diploma (no academic degree) } \\
\hline Bachelor student & 2.73 & 0.936 & 2.44 & 0.844 & 1.02 & 0.964 \\
\hline Bachelor diploma & 1.54 & 0.996 & 1.58 & 0.977 & 0.40 & 1.000 \\
\hline Master student & -1.49 & 0.999 & -0.97 & 0.999 & -0.13 & 1.000 \\
\hline Master diploma & -0.19 & 1.000 & 0.07 & 1.000 & 0.37 & 1.000 \\
\hline $\mathrm{PhD} /$ doctorate student & -2.65 & 0.978 & -1.38 & 0.996 & -0.37 & 1.000 \\
\hline $\mathrm{PhD} /$ doctorate diploma & 3.73 & 0.905 & 2.24 & 0.961 & 1.82 & 0.825 \\
\hline \multicolumn{7}{|l|}{ Bachelor student } \\
\hline Bachelor diploma & -1.20 & 0.809 & -0.86 & 0.820 & -0.62 & 0.610 \\
\hline Master student & -4.22 & 0.118 & -3.41 & 0.055 & -1.15 & 0.616 \\
\hline Master diploma & -2.92 & 0.104 & -2.37 & 0.044 & -0.65 & 0.793 \\
\hline $\mathrm{PhD} /$ doctorate student & -5.39 & 0.102 & -3.82 & 0.120 & -1.39 & 0.655 \\
\hline $\mathrm{PhD} /$ doctorate diploma & 1.00 & 0.999 & -0.20 & 1.000 & 0.80 & 0.975 \\
\hline \multicolumn{7}{|l|}{ Bachelor diploma } \\
\hline Master student & -3.02 & 0.424 & -2.55 & 0.242 & -0.53 & 0.982 \\
\hline Master diploma & -1.72 & 0.554 & -1.52 & 0.314 & -0.03 & 1.000 \\
\hline $\mathrm{PhD} /$ doctorate student & -4.19 & 0.317 & -2.97 & 0.351 & -0.76 & 0.966 \\
\hline $\mathrm{PhD} /$ doctorate diploma & 2.20 & 0.942 & 0.65 & 1.000 & 1.42 & 0.675 \\
\hline \multicolumn{7}{|l|}{ Master student } \\
\hline Master diploma & 1.30 & 0.987 & 1.03 & 0.979 & 0.50 & 0.992 \\
\hline $\mathrm{PhD} /$ doctorate student & -1.17 & 0.999 & -0.42 & 1.000 & -0.24 & 1.000 \\
\hline $\mathrm{PhD} /$ doctorate diploma & 5.22 & 0.358 & 3.21 & 0.571 & 1.95 & 0.510 \\
\hline \multicolumn{7}{|l|}{ Master diploma } \\
\hline $\mathrm{PhD/doctorate} \mathrm{student}$ & -2.46 & 0.893 & -1.45 & 0.960 & -0.74 & 0.979 \\
\hline $\mathrm{PhD} /$ doctorate diploma & 3.92 & 0.559 & 2.17 & 0.824 & 1.45 & 0.704 \\
\hline \multicolumn{7}{|l|}{ PhD/doctorate student } \\
\hline $\mathrm{PhD} /$ doctorate diploma & 6.39 & 0.241 & 3.62 & 0.549 & 2.19 & 0.497 \\
\hline
\end{tabular}

${ }^{a}$ CAEB: Connotative Aspects of Epistemological Beliefs.

${ }^{\mathrm{b}} \mathrm{MD}$ : mean difference; Sig: significance ( $p$ value). 
No statistical differences were found in the general characteristics for years licenced

Table 10. MANOVA test results for years licenced.

\begin{tabular}{|c|c|c|c|c|c|c|}
\hline & \multicolumn{2}{|c|}{ CAEB $^{\mathrm{a}}$-total } & \multicolumn{2}{|c|}{ CAEB-Text } & \multicolumn{2}{|c|}{ CAEB-Variab } \\
\hline & $\mathrm{MD}^{\mathrm{b}}$ & $\mathrm{Sig}^{\mathrm{b}}$ & MD & Sig & MD & Sig \\
\hline \multicolumn{7}{|l|}{0} \\
\hline$<5$ & -4.44 & 0.840 & -0.65 & 1.000 & -2.88 & 0.495 \\
\hline $5-10$ & -4.22 & 0.867 & -0.93 & 0.998 & -2.46 & 0.656 \\
\hline $11-15$ & -3.23 & 0.947 & -0.14 & 1.000 & -2.00 & 0.812 \\
\hline$>15$ & -4.55 & 0.828 & -0.91 & 0.998 & -2.84 & 0.512 \\
\hline \multicolumn{7}{|l|}{$<5$} \\
\hline $5-10$ & 0.22 & 0.999 & -0.28 & 0.995 & 0.43 & 0.825 \\
\hline $11-15$ & 1.21 & 0.836 & 0.52 & 0.973 & 0.89 & 0.359 \\
\hline$>15$ & -0.11 & 1.000 & -0.26 & 0.993 & 0.04 & 1.000 \\
\hline \multicolumn{7}{|l|}{$5-10$} \\
\hline $11-15$ & 0.98 & 0.940 & 0.79 & 0.915 & 0.46 & 0.913 \\
\hline$>15$ & -0.33 & 0.997 & 0.02 & 1.000 & -0.38 & 0.887 \\
\hline \multicolumn{7}{|l|}{$11-15$} \\
\hline$>15$ & -1.31 & 0.802 & -0.77 & 0.899 & -0.84 & 0.434 \\
\hline
\end{tabular}

${ }^{a}$ CAEB: Connotative Aspects of Epistemological Beliefs.

${ }^{\mathrm{b}} \mathrm{MD}$ : mean difference; Sig: significance.

\section{Discussion}

\section{Epistemic literacy in physiotherapists}

This study aims to explore the level of sophistication in domain-specific epistemic beliefs in physiotherapy within Europe.

The low to moderate epistemic sophistication is in line with the criticism regarding the evidence-based practice movement [12,19]. In this movement, knowledge is still seen as objective, context-free scientific facts, predominantly derived from empirical-analytical research [12,19]. Such knowledge can then be 'translated' into the explicit and rational decision making of clinicians.

This is in contrast with the assumed complex and uncertain nature of practice. In this sense, evidence is always a situation-based, negotiated product [20]. Many sources of evidence, such as client values and goals, scientific evidence and experiential evidence, are used. Formal knowledge is melted down into a mixture of the tacit and explicit practical knowledge that works best. This puts demands on practitioners. Epistemic sophistication could offer an opportunity to improve the critical use of different resources in evidence-based practice [12].

We performed a multiple level comparison and therefore the likelihood for Type 1 errors is increasing. No correction was used in this study and therefore we show all outcomes in Tables $7-$ 10 [21]. In the interpretation of the results, we have to be aware that especially the (low) significant differences measured in the levels of education can only be regarded as tentative. We discuss these results accordingly. 
The results of this study show no significant increase of the epistemic sophistication with a higher level of education. This is similar to the results of the only other study measuring the epistemic beliefs in physiotherapists [7]. This study of Bientzle et al. shows also no significant difference in sophistication of epistemic beliefs between advanced students and professionals [7]. According to our study, sophistication of epistemic beliefs seems to be more related to with age than to the level of education. For the factor variability, the sophistication of epistemic beliefs increases with age. So, knowledge is perceived to be more flexible and dynamic when people become older.

It would be interesting to research whether the amount of real contact with patient increases sophistication. The underlying argument to this question is that real patient contact confronts the practitioner with the complexity of practice.

We infer that in the European community of physiotherapy countries with similar epistemic beliefs exist. Based on this inference, it seems relevant to further jointly develop the framework and target interventions within evidence-based practice.

More research should be done into the differences shown between the different regions in Europe. This could offer better understanding of these differences and could help to design the collaboration between countries in the common effort to improve evidence-based practice.

\section{Validity and usability of the $C A E B$}

The results show a valid CAEB-questionnaire for the different languages from this sample. This offers an opportunity of using this questionnaire to measure the effect of interventions in order to increase the level of sophistication within the field of physiotherapy and to make comparisons between the countries. The associative-evaluative nature of the CAEB makes it especially suitable for the complexity of evidence-based physiotherapy practice.

\section{Limitations}

The researchers were only able to get results from a selection of countries in Europe. The comparable results within the same region of Europe would expect similar results from other countries in that region, though this should be further researched. Unfortunately, the East European countries are missing.

Earlier, we mentioned the risks in performing a multiple level comparison. In order to see if significant differences between groups exist, more studies with high power need to be done. Also some of the groups in the sample turn out to be small. This jeopardises the statistical power. This is the case with the subgroup Sweden. Also, the groups 'level of educational, professional diploma, $\mathrm{PhD}$ and $\mathrm{PhD}$ candidates' are small.

\section{Implications}

In order to work evidence-based within the inherent complexity of the profession, a high level of sophistication in epistemic beliefs is a prerequisite. The results of this study indicate that the sophistication in epistemic beliefs can improve significantly in the community of physiotherapists in Europe. The process starts with a firm attention to epistemic beliefs in physiotherapy curricula. This study shows that it could beneficial to create a joint European endeavour to improve education in this field.

The assumption is that an increase in sophistication will help the evidence-based practice movement to overcome its challenges $[12,19]$. Even so it will scrutinise the epistemic premises of the framework of evidence-based practice and its tools $[12,19]$. 
More research needs to be done in the field of epistemic beliefs of physiotherapists; methodology needs to be improved both for measuring the concept and for intervention.

It seems advisable to focus on sophisticated epistemic beliefs in the early phases of physiotherapy education, thus preparing for the context-specificity and dynamics of knowledge in daily practice. Besides physiotherapy education, an increase in sophistication of epistemic beliefs among practitioners seems also paramount for improving evidence-based practice [22,23].

\section{Disclosure statement}

The authors have declared that there are no potential conflicts of interest with respect to the authorship and/or publication of this article. This study has no funding resource and follows the principles of the Declaration of Helsinki with regard to ethical considerations. This study and manuscript have not been previously presented or published elsewhere.

\section{References}

[1] Hofer B. Dimensionality and disciplinary differences in personal epistemology. Contemporary Educational Psychology. 2000;25:378-405.

[2] Hofer BKG. Personal epistemology and culture. In: Khine MS, editor. Knowing, knowledge and beliefs. Dordrecht: Springer; 2008.

[3] King PM, Kitchener KS. Developing reflective judgment: understanding and promoting intellectual growth and critical thinking in adolescents and adults. San Francisco, CA: Jossey-Bass; 1994.

[4] Hofer BK, Sinatra GM. Epistemology, metacognition, and self-regulation: musings on an emerging field. Metacogn Learn. 2010;5:113-120.

[5] Roex A, Clarebout G, Dory V, et al. Can ill-structured problems reveal beliefs about medical knowledge and knowing? A focus-group approach. BMC Med Educ. 2009;9:62.

[6] Otting H, Zwaal W, Tempelaar D, et al. The structural relationship between students' epistemological beliefs and conceptions of teaching and learning. Stud High Educ. 2010;35:741-760.

[7] Bientzle M, Cress U, Kimmerle J. Epistemological beliefs and therapeutic health concepts of physiotherapy students and professionals. BMC Med Educ. 2014;14:208.

[8] Stahl E, Bromme R. The CAEB: an instrument for measuring connotative aspects of epistemological beliefs. Learn Instruct. 2007;17:773-785.

[9] Clarke CL, Wilcockson J. Seeing need and developing care: exploring knowledge for and from practice. Int J Nurs Stud. 2002;39:397-406.

[10] Green HJ, Hood M. Significance of epistemological beliefs for teaching and learning psychology: a review. Psychol Learn Teach. 2013;12:168-178.

[11] Kienhues D, Bromme R, Stahl E. Changing epistemological beliefs: the unexpected impact of a short-term intervention. Br J Educ Psychol. 2008;78:545-565.

[12] Greenhalgh T, Howick J, Maskrey N. Evidence based medicine: a movement in crisis? BMJ. 2014;348:g3725.

[13] Louca L, Elby A, Hammer D, et al. Epistemological resources: applying a new epistemological framework to science instruction. Educ Psychol. 2004;39:57-68.

[14] Baxter Magolda MB. Evolution of a constructivist conceptualization of epistemological reflection. Educ Psychol. 2004;39:31-43.

[15] Stehr N. Knowledge societies. London: Sage; 1994.

[16] Beenen PC, Filiputti D, Meyer ER, et al. Epistemological beliefs of European physiotherapists - a multi-country cross-cultural adaptation for the DEBQ and the CAEB questionnaires. Eur J Physiother. 2016;11:32-39.

[17] Comrey AL, Lee HB. A first course in factor analysis. 2nd ed. Hillsdale (NJ): Lawrence Erlbaum Associates; 1992.

[18] Hair JF, Anderson RE, Tatham RL, et al. Multivariate data analysis. 5th ed. Upper Saddle River (NJ): Prentice-Hill; 1998.

[19] Marks DF. Perspectives on evidence-based practice. Health Development Agency Public Health Evidence Steering Group (02); 2002. p. 1-53.

[20] Wieringa S, Greenhalgh T. 10 years of mindlines: a systematic review and commentary. Implement Sci. 2015;10:45.

[21] Feise RJ. Do multiple outcome measures require p-value adjustment? BMC Med Res Methodol. 2002;2:81471-82288. 
[22] Dall'Alba G. Understanding professional practice: investigations before and after an educational programme. Stud High Educ. 2004;29:679-692.

[23] Dall'Alba G, Sandberg J. Unveiling professional development: a critical review of stage models. Rev Educ Res. 2006;76:383-412. 